\title{
Analysis of Thrombosis Risk Stratification Models Based on 10 Years Follow up of 237 Essential Thrombocythemia Patients
}

ISSN: 2637-773X

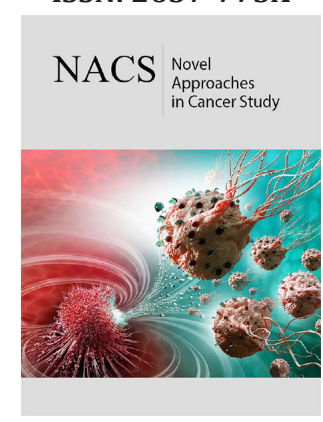

*Corresponding author: Miklos Egyed, Department of Haematology, Tallián Gyulautca 20-32, Kaposvár, 7400, Hungary

Submission: 眥 November 20, 2020

Published: 嗹January 22, 2021

Volume 5 - Issue 5

How to cite this article: Adam Kellner Vasana Kellner, Peter Rajnics, Eva Karádi, Miklos Egyed, et al. Analysis of Thrombosis Risk Stratification Models Based on 10 Years Follow up of 237 Essential Thrombocythemia Patients. Nov Appro in Can Study. 5(5). NACS.000621. 2021. DOI: 10.31031/NACS.2021.05.000621

Copyright@ Miklos Egyed, This article is distributed under the terms of the Creative Commons Attribution 4.0 International License, which permits unrestricted use and redistribution provided that the original author and source are credited.
Adam Kellner ${ }^{1}$, Vasana Kellner ${ }^{1}$, Peter Rajnics ${ }^{1}$, Eva Karádi ${ }^{1}$, Arpad Illes ${ }^{2}$, Miklos Udvardy², Lajos Homor ${ }^{3}$, Peter Dombi ${ }^{4}$, Jozsef Herczeg ${ }^{1}$, Zoltan Sipiczki ${ }^{5}$, Viktoria Gyorine Korom ${ }^{1}$, and Miklos Egyed ${ }^{1}$

${ }^{1}$ Department of Hematology, Hungary

${ }^{2}$ Department of Hematology, Hungary

${ }^{3}$ Faculty of Humanities and Social Sciences, Hungary

${ }^{4}$ Department of Hematology, Hungary

\begin{abstract}
Objective: In our study we analysed thromboembolic (TE) events of ET patients using data from the Hungarian Myeloproliferative Neoplasm (HUMYPRON) registry. We examined possible thrombosis risk factors and compared Landolfi-, IPSET- and R-IPSET risk stratification models.
\end{abstract}

Methods: The HUMYPRON registry was estabilished in 2012 and contains data of patients from 14 hungarian haematology centers. We analysed clinical and laboratory data of 237 ET (according to 2008 WHO classification) patients to assess possible risk factors of post-diagnostic TE events and to study the applicability of Landolfi-, IPSET- and R-IPSET thrombosis risk stratifications.

Results: 237 ET patients were followed for 10 years on average. After diagnosis 76 patient (32.1\%) had TE complication. Previous thrombotic episode was found to be the only factor that had significant effect on TE events after diagnosis $(\mathrm{p}<0.001)$. There were marginally less TE events after diagnosis in case of patients who had extremely high $(\geq 1.000 \times 109 / \mathrm{L})$ platelet count measured at the time of diagnosis ( $p=0.047$ ). On our sample R-IPSET proved to be the strongest model, where the frequency of TE events was $14.3 \%$ in the very low and $46 \%$ in the high-risk patient group.

Conclusions: During the follow-up period of 10 years on average, prior TE event was the only factor that had highly significant effect on post-diagnostic thrombosis. Among the risk stratification systems R-IPSET model proved to be the strongest.

Keywords: Essential thrombocythaemia;Thrombosis;Humypron registry;Risk factors; Landolfi; IPSET; R-IPSET

Abbreviations: AMIN:Minor Arterial TE Event; AMAJ: Major Arterial TE Event; VMAJ:Major Venous TE Event; ANA:Anagrelide; HU:Hydroxyurea; ASA:Acetylsalicylic acid

\section{Introduction}

Essential Thrombocythaemia (ET) is a clonal stem cell disease that belongs to the Philadelphia Negative Chronic Myeloproliferative Neoplasms (MPN) and is characterized by megakaryocytosis of the bone marrow and thrombocytosis. The incidence of the disease in western European countries is $0.2-2.5 / 100,000$ people, its prevalence is $38-57 / 100,000$ $[1,2]$. The average age at diagnosis is 60 years, and the incidence is twice as high in women than in men [3]. The diagnosis of ET is currently established according to 2016 WHO criteria $[4,5]$. There is no specific molecular or genetic marker for diagnosis of the disease. The most common alteration is the presence of Janus kinase 2 (JAK2) V617F activation mutation, which occurs in about $50-60 \%$ of the cases [5-7]. Calreticulin (CALR) mutation is present in $15-32 \%$ of patients, and myeloproliferative leukemia virus oncogene (MPL) mutation appears in 3-4\% [5-7]. $10-20 \%$ of all cases is triple negative, where none of these mutations can be detected [6-8].

The median survival for the entire patient population is 20 years, but for those younger than 60 this value is already 30 years [8]. Morbidity and mortality of patients are primarily determined by thrombotic and haemorrhagic events. There are significant differences in the incidence of thrombohaemorrhagic events in the literature, which may be resulted by 
differences in event definition, patient selection, and applied therapy. In a study of 100 patients, thrombotic events occurred at a rate of $6.6 \% /$ patient-year and haemorrhagic events at a frequency of $0.33 \% /$ patient-year [9]. In another report, the incidences of thrombotic and bleeding events were $8.1 \%$ patientyear and $2.5 \% /$ patient-year respectively [10]. Transformation into myelofibrosis occurs in $0.8-4.9 \%$ in 10 years and $4-11 \%$ in 15 years [8]. Transformation into acute myeloid leukaemia is observed in $0.7-3 \%$ in 10 years and $2.1-5.3 \%$ in 15 years [8].

Because of the very good patient survival and the lack of evidence for life prolongation of any kind of medication, recent therapy of ET focuses on prevention of the thrombo hemorrhagic events. Identification of risk groups and their appropriate management are highly important tasks. There are several risk stratifications for thrombosis. The IPSET-thrombosis (International Prognostic Score for Thrombosis in Essential Thrombocythemia) risk stratification takes age, previous thrombosis, JAK2 positivity and cardiovascular risk factors into account [11]. In this score system, the pre-diagnostic thrombosis and JAK2 positivity are more heavily weighted. Landolfi categorizes patients into low, medium, high, and extreme high-risk groups based on age, pre-thrombotic events, white blood cell and platelet count, and general vascular risk factors [12]. In his therapeutical recommendation, acetylsalicylate (ASA) treatment is sufficient for patients at low to moderate risk for thrombosis, whereas cytoreductive therapy is recommended for patients at high and extreme risk.

The revised IPSET model for thrombosis (R-IPSET) proposed by Tefferi distinguishes four groups, very low, low, medium, and high risk, based on age, prior TE event, and JAK2/MPL mutation [13]. Cardiovascular (CV) risk factors are not included in his risk assessment of thrombosis but are taken into account at the therapeutic recommendation. Accordingly, it is not necessary to treat patients at very low risk for thrombosis who do not have a cardiovascular risk factor. While, in presence of CV risk factor he recommends 1x100 mg ASA per day. Low-risk patients are advised to take $1 \times 100 \mathrm{mg}$ or $2 \times 100 \mathrm{mg}$ ASA daily depending on CV risk factors. He warns of giving ASA for those with an extremely high platelet count $\left(\geq 1.000 \times 10^{9} / \mathrm{l}\right)$ due to increased bleeding tendency. For patients at moderate risk of thrombosis who have no CV risk factor, $1 \times 100 \mathrm{mg}$ ASA is suggested on a daily basis, while in the presence of CV risk factor hydroxyurea is needed in addition to ASA. Patients at high risk for thrombosis, depending on whether they had an arterial or venous TE event, are recommended to receive $2 \times 100 \mathrm{mg}$ ASA or systemic anticoagulation in addition to hydroxyurea.

Landolfi and Tefferi both attribute significant importance to previous TE events, and when they occur, the patient is classified into high-risk category. The Hungarian MPN Working Group (HUMYPRON GROUP) is an online registry that contains clinical and laboratory data of patients with myeloproliferative neoplasms $[14,15]$. In our study we compared the usability of these three risk stratifications on the ET patient group of the HUMYPRON database with a follow up period of 10 years.

\section{Methods}

\section{Subjects and data collection}

The HUMYPRON registry was founded in 2012 and contains data of 237 ET patients from 14 hungarian haematology centers. The diagnosis of ET was established according to the 2008 World Health Organization criteria. The details of study methods have been published before [14]. In brief, clinicians completed a questionnaire that focuses on risk stratification, treatment and complications. As for thrombotic risk stratification Landolfi-, IPSET- and R-IPSET scoring systems were used. Data of ET patients was analysed retrospectively with a cut-off date of December 2018. Based on the database we examined potential risk factors of thrombosis and compared Landolfi-, IPSET- and R-IPSET risk stratification systems. The aim of the present study was to determine the risk stratification that proved to be the most useful when examining this group of patients.

\section{Definition of thrombotic events}

Thrombotic events were defined according to Gisslinger [16]:

1. Major arterial thrombosis: stroke, myocardial infarction, peripheral arterial thrombosis and splanchnic arterial thromboembolism.

2. Minor arterial thrombosis: TIA, angina pectoris, unstable angina, generalized convulsion, erythromelalgia, ocular symptoms, angina abdominalis (transient abdominal ischemia).

3. Major venous events: deep venous thrombosis, pulmonary embolism, splanchnic venous thrombosis, and other major venous events.

Minor venous events were not considered in this study. When a patient suffered more thromboembolic episodes of the same phenotype, we only regarded it once.

\section{Treatment}

ET patients of our study received either anagrelide or hydroxiurea+acetylsalicilic acid therapy. Those who switched from one type of medication to the other were excluded from the analysis. Because of platelet aggregation inhibition caused by the blocking of phosphodiestherase type III. enzyme patients who were treated with anagrelide did not get arterial thromboprophylaxis. For those who had already been anticoagulated due to prior major venous TE event we continued venous thromboprophylaxis. Because of the retrospective and" real life" nature of our analysis, involving 14 haematology centres, therapeutic decisions were made by the clinicians, not necessarily according to the same protocoll. Conclusions regarding therapy have been published previously [17]. 


\section{Statistical analysis}

We used Kolmogorov- Smirnov test to determine the distribution of continuous variables (type I error $=10 \%$ ). Samples showing normal distribution were compared with $t$ test for independent samples (test for variance homogeneity: Levene test, type I error $=5 \%$ ). In other cases, the Mann-Whitney U test with exact probabilities was carried out. Ordinal variables were also compared using the Mann-Whitney U test. We used Fisher's exact test or the exact chi-squared $\left(\chi^{2}\right)$ test to analyse categorical variables.

Logistic regression analysis (forward method) was used to investigate how treatment (anagrelide vs hydroxyurea+aspirin), age at diagnosis ( $<60$ years vs. $>60$ years), JAK2 ${ }^{\mathrm{V} 617 \mathrm{~F}}$ positivity (yes or no), gender (male vs. female) and pre-diagnostic thromboembolism (yes or no) influenced post-diagnostic TE events.

Since type I error was not adjusted for multiple testing our results are only descriptive. Statistical analysis was performed using the open-source R statistical software package, version 3.1.2.
Statistical tests were evaluated at a significance level of 5\%.

\section{Ethics and study management}

The test was carried out under license from ETT-TUKEB in compliance with the principles of GCP and Helsinki Declaration. Patients gave written consent to use their data anonymously after being informed of the nature of the study.

\section{Result}

\section{Patient characteristics and thrombotic risk}

We analysed the data of $237 \mathrm{ET}$ patients with an average followup of 10 years (range from 1-29 years). The mean age at diagnosis was 60.9 years. Female predominance was observed with a male: female ratio of $1: 2$. JAK2V617F mutation positivity was present in $70.5 \%$ of the cases. 116 patients received anagrelid therapy, 121 patients were treated with the combination of hydroxiurea and acetylsalcylic acid. Our findings about different treatments, the effect of medication on thrombotic risk, disease progression and survival have been published earlier [17].

\section{Thrombotic events}

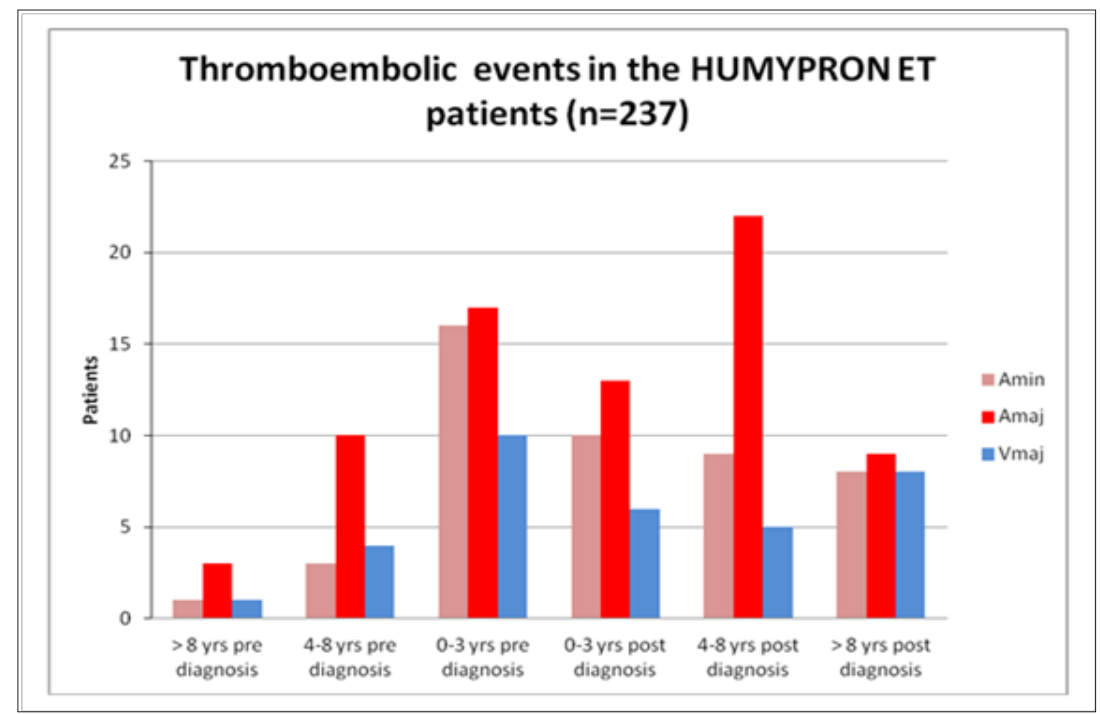

Figure 1:Occurrence of thromboembolic events before the diagnosis and during follow up.

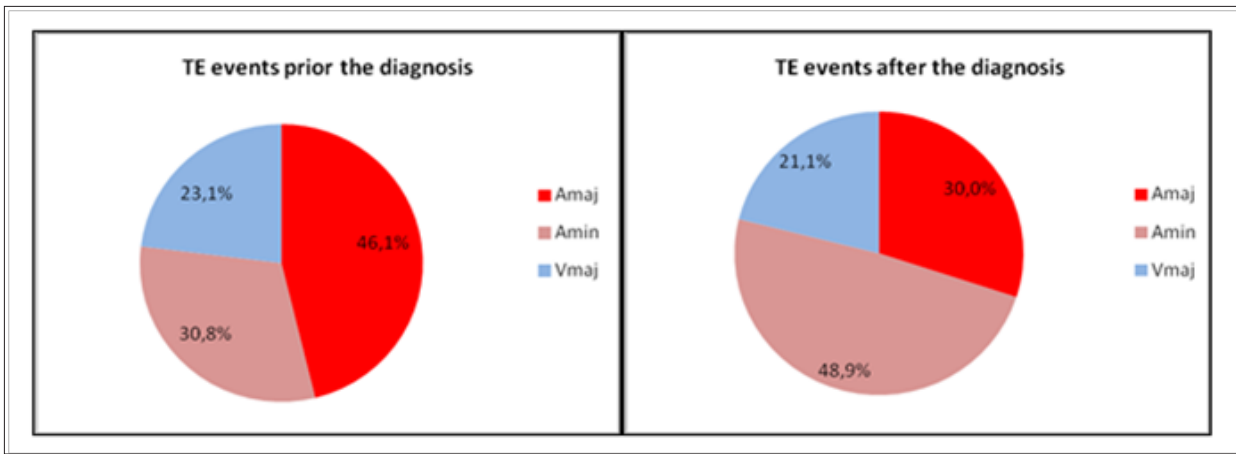

Figure 2:Distribution of thromboembolic events before and after the diagnosis. 
Before the diagnosis 63 patients $(26.6 \%)$ had 65 thromboembolic events. The earliest TE event occurred 10 years before diagnosis (Figure 1). Major arterial thrombosis was the most common complication $(n=30,46.1 \%)$. Minor arterial thrombosis was registered in 20 cases $(30.8 \%)$, and we found major venous TE in 15 cases (23.1\%). After the diagnosis 76 patients $(32.1 \%)$ suffered 90 TE complications. The latest TE episode was registered 19 years after the diagnosis. Minor arterial events being the most common ( $\mathrm{n}=44,48.9 \%)$. Major arterial TE was observed in 27 (30.0\%) and major venous TE in 19 (21.1\%) cases (Figure 2). Minor venous events were not taken into account during the follow- up period of 10 years on average. Bleeding complication occured in case of 8 patients $(3.4 \%)$.

Those who had thrombotic event prior to ET diagnosis were significantly more likely to have a thrombotic event after diagnosis ( $p$ <.001). Prior thrombotic event turned out to be independent risk factor. At the same time, no significant correlation was found with any other parameter, either age, diagnostic blood count, or mutation status (Table 1). Logistic regression analysis (multivariate model) indicated that only TE prior to diagnosis influence the TE incidence significantly $(\mathrm{p}<0.001$; Table 2$)$.

Table 1: Characteristics of patients with ET $(n=237)$ and occurrence of thromboembolic events.

\begin{tabular}{|c|c|c|c|c|}
\hline & TE, yes, n (\%) & TE, no, n (\%) & \multirow{2}{*}{ OR (CI 95\%) } & \multirow{2}{*}{$\mathbf{p}$} \\
\hline & $\mathrm{N}=76$ & $N=161$ & & \\
\hline \multicolumn{3}{|l|}{ Gender } & \multirow{3}{*}{$0.860(0.477-1.549)$} & \multirow{3}{*}{0.615} \\
\hline Male, n (\%) & $23(30.3)$ & $54(33.5)$ & & \\
\hline Female, n (\%) & $53(69.7)$ & $107(66.5)$ & & \\
\hline \multicolumn{3}{|c|}{ Age at diagnosis categorised, years } & \multirow{3}{*}{$0.806(0.463-1.404)$} & \multirow{3}{*}{0.447} \\
\hline <60 years, $\mathrm{n}(\%)$ & $30(39.5)$ & $72(44.7)$ & & \\
\hline$\geq 60$ years, $\mathrm{n}(\%)$ & $46(60.5)$ & $89(55.3)$ & & \\
\hline \multicolumn{3}{|l|}{ TE prior to diagnosis } & \multirow{3}{*}{$4.926(2.663-9.114)$} & \multirow{3}{*}{$<0.0001$} \\
\hline yes, n (\%) & $37(48.7)$ & $26(16.1)$ & & \\
\hline no, n (\%) & $39(51.3)$ & $135(83.9)$ & & \\
\hline \multicolumn{3}{|l|}{ JAK2 status } & \multirow{3}{*}{$1.703(0.906-3.204)$} & \multirow{3}{*}{0.099} \\
\hline positive, n (\%) & $59(77.6)$ & $108(67.1)$ & & \\
\hline negative, n (\%) & $17(22.4)$ & $53(32.9)$ & & \\
\hline \multicolumn{3}{|l|}{ CALR status } & \multirow{3}{*}{$0.546(0.254-1.170)$} & \multirow{3}{*}{0.12} \\
\hline positive, n (\%) & $10(13.2)$ & $35(21.7)$ & & \\
\hline negative, n (\%) & $66(86.8)$ & $126(78.3)$ & & \\
\hline \multicolumn{3}{|l|}{ MPL status } & \multirow{3}{*}{$1.549(0.475-5.050)$} & \multirow{3}{*}{0.468} \\
\hline positive, n (\%) & $5(6.6)$ & $7(4.3)$ & & \\
\hline negative, n (\%) & $71(93.4)$ & $154(95.7)$ & & \\
\hline \multicolumn{3}{|l|}{$\mathrm{WBC}$} & \multirow{3}{*}{$0.800(0.426-1.504)$} & \multirow{3}{*}{0.488} \\
\hline$\geq 10.000, \mathrm{n}(\%)$ & $18(23.7)$ & $45(28.0)$ & & \\
\hline <10.000, n (\%) & $58(76.3)$ & $116(72.0)$ & & \\
\hline \multicolumn{3}{|l|}{ PLT } & \multirow{3}{*}{$0.480(0.231-0.994)$} & \multirow{3}{*}{0.048} \\
\hline$\geq 1.000 .000, \mathrm{n}(\%)$ & $11(20,8)$ & $42(79,2)$ & & \\
\hline$<1.000 .000, \mathrm{n}(\%)$ & $65(35,3)$ & $119(64,7)$ & & \\
\hline \multicolumn{3}{|l|}{$\mathrm{MCH}$} & \multirow{3}{*}{$1.639(0.757-3.550)$} & \multirow{3}{*}{0.21} \\
\hline <28pg, n (\%) & 13 (17.1) & $18(11.2)$ & & \\
\hline 228pg, n (\%) & 63 (82.9) & $143(88.8)$ & & \\
\hline \multicolumn{3}{|l|}{ LDH } & \multirow{3}{*}{$0.800(0.395-1.620)$} & \\
\hline high, n (\%) & $24(26,7)$ & $66(73,3)$ & & 0.535 \\
\hline not high, n (\%) & $20(31,3)$ & $44(68,7)$ & & \\
\hline
\end{tabular}




\begin{tabular}{|c|c|c|c|c|}
\hline Diabetes & & & \multirow{3}{*}{$0.957(0.430-2.137)$} & \multirow{3}{*}{0.915} \\
\hline yes, n (\%) & $10(31,2)$ & $22(68,8)$ & & \\
\hline no, n (\%) & $66(32,2)$ & $139(67,8)$ & & \\
\hline Dyslipidaemic & & & \multirow{3}{*}{$0.753(0.402-1.411)$} & \multirow{3}{*}{0.376} \\
\hline yes, n (\%) & $18(27,7)$ & $47(72,3)$ & & \\
\hline no, n (\%) & $58(33,7)$ & $114(66,3)$ & & \\
\hline Hypertonia & & & \multirow{3}{*}{$1.180(0.677-2.056)$} & \multirow{3}{*}{0.56} \\
\hline yes, n (\%) & $46(33,6)$ & $91(66,4)$ & & \\
\hline no, n (\%) & $30(30,0)$ & $70(70,0)$ & & \\
\hline
\end{tabular}

Table 2: Influence of different patient characteristics on post-diagnostic TE complications (total $\mathrm{n}=237$ ), logistic regression (multivariate model).

\begin{tabular}{|c|c|c|c|}
\hline Variables & $\mathbf{p}$ & $\operatorname{Exp}(B):$ hazards ratio & $\operatorname{Exp}(B): 95 \% \mathrm{CI}$ \\
\hline Treatment (ANA vs. HU+ASA) & 0.114 & 1,641 & $0,888-3,031$ \\
\hline Age at the diagnosis (years) & 0,298 & 1,016 & $0,986-1,048$ \\
\hline Gender (male vs. female) & 0,771 & 0,908 & $0,473-1,741$ \\
\hline JAK2 mutation (yes vs. no) & 0,115 & 1,733 & $0,874-3,437$ \\
\hline TE event before the diagnosis (yes vs. no) & $<0,001$ & 6,130 & $2,897-12,972$ \\
\hline Time to death/length of follow-up (years) & 0,903 & 1,004 & $0.944-1,067$ \\
\hline
\end{tabular}

\section{Thrombotic risk stratifications}

We applied and analysed Landolfi-, IPSET- and R-IPSET risk stratifications in 237 ET patients of the „HUMYPRON” registry. In Landolfi's low risk group, $37.5 \%$ of patients had a thrombotic event after diagnosis, whereas in the very high-risk group thrombotic events were registered in $44.9 \%$ of the cases. At the same time the proportion of TE events in Landolfi's low risk group (37.5\%) was higher than what we observed in the medium $(23.8 \%)$ and high risk $(27.8 \%)$ groups (Table 3; Figure 3). According to the IPSET thrombosis model $18.8 \%$ of the patients in the low-risk group suffered TE complication, while in the high-risk group this proportion was $39.0 \%$. In the intermediate group fewer thrombotic events were registered than in the low-risk group (Table 3; Figure 4).

Table 3: Occurrence of thromboembolic events according to risk categories in Landolfi-, IPSET- and R-IPSET models.

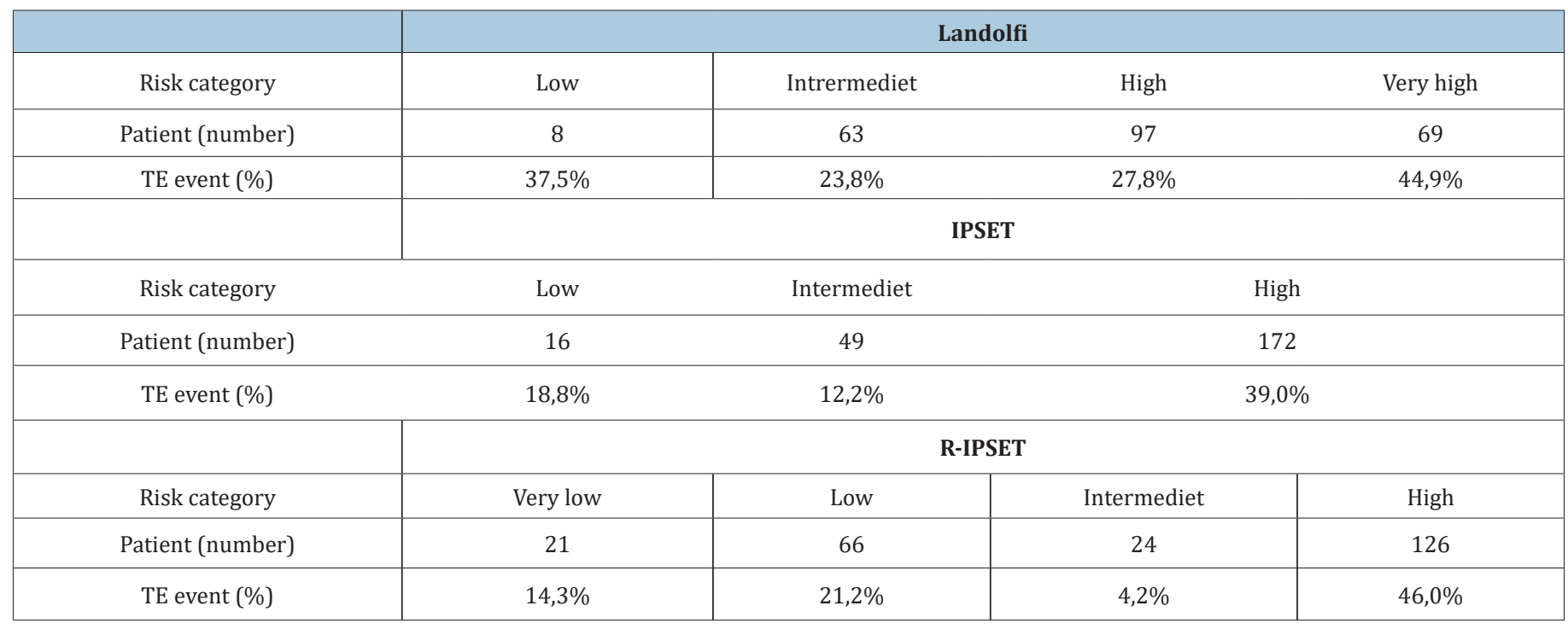




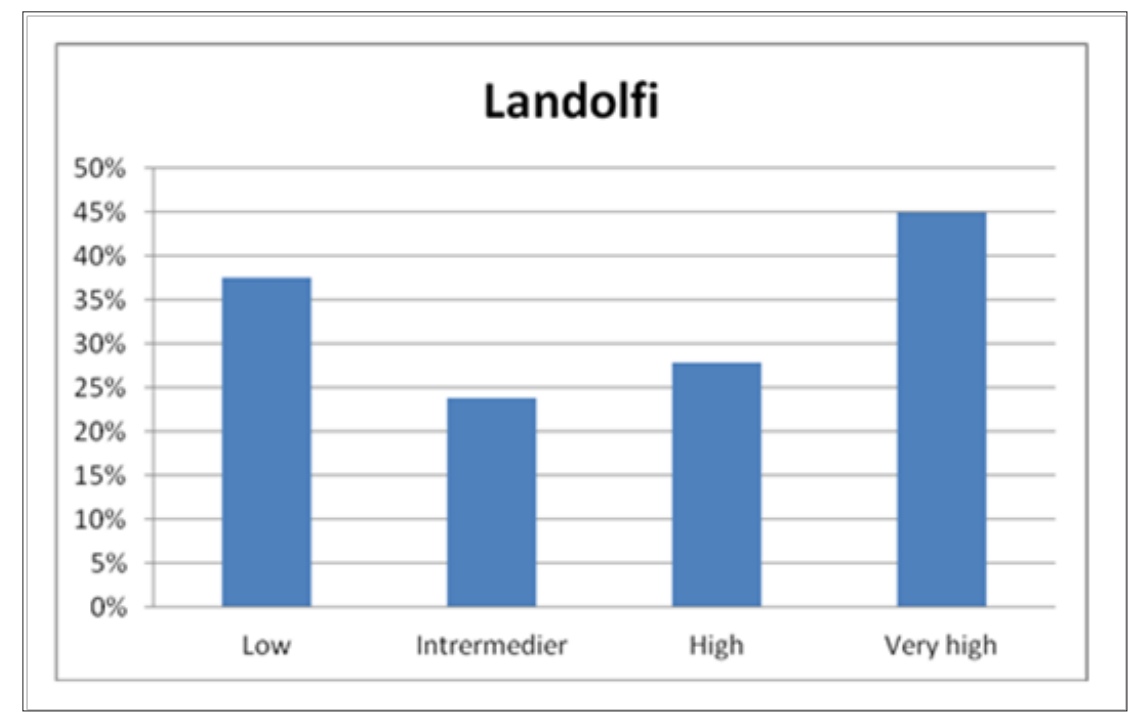

Figure 3:Occurrence of thromboembolic events according to Landolfi thrombosis risk model.

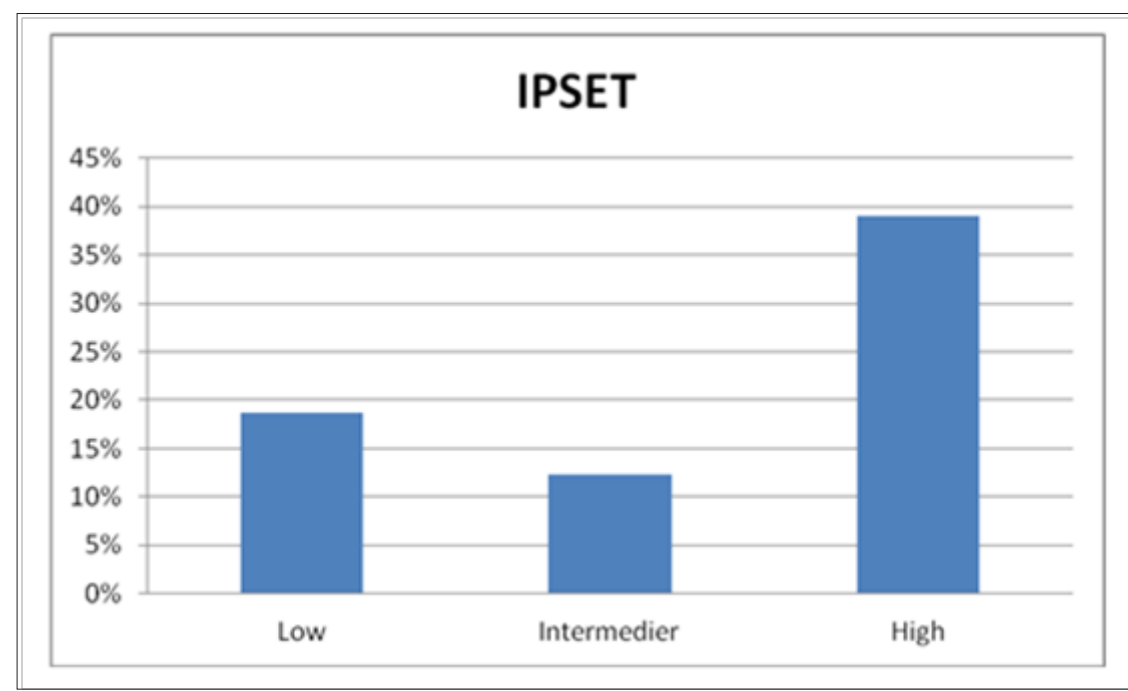

Figure 4: Occurrence of thromboembolic events according to IPSET thrombosis risk model.

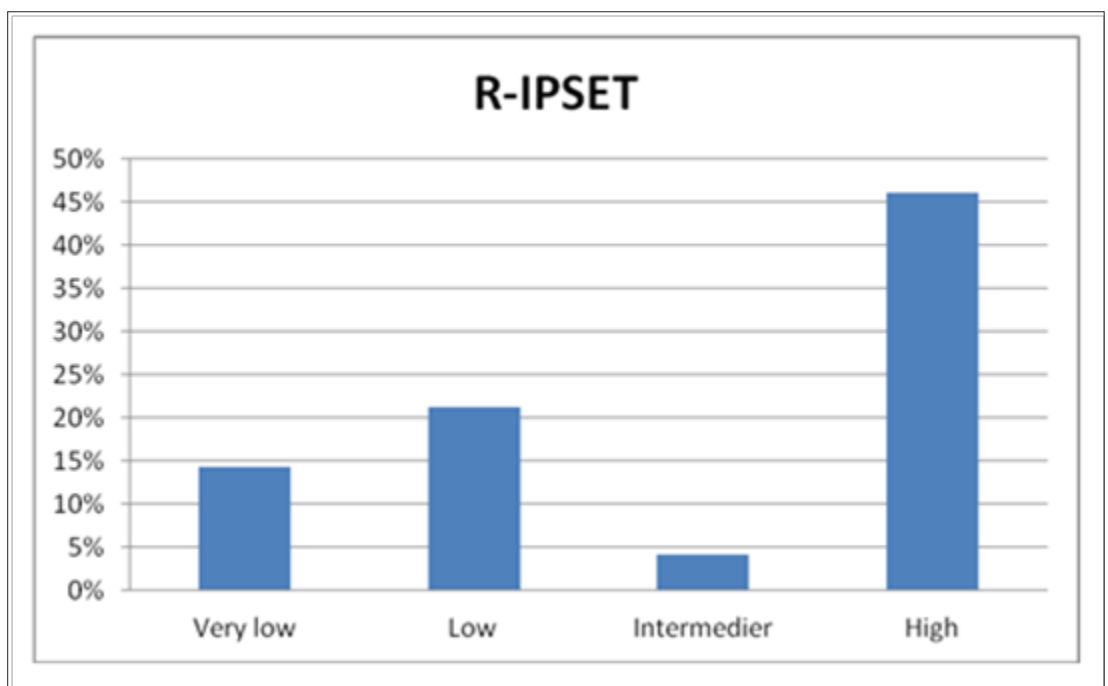

Figure 5: Occurrence of thromboembolic events according to R-IPSET thrombosis risk model. 
As for the R-IPSET risk stratification, $14.3 \%$ of patients of the very low risk group suffered thrombosis after diagnosis, whereas this ratio was $46.0 \%$ amongst high-risk group patients. In the intermediate risk group, however, TE events were registered with lower frequency than in the prior low-, and very low risk categories (Table 3; Figure 5).

Comparing all categories, it was the R-IPSET high risk group in which the highest proportion of thrombotic events was observed after diagnosis (46.0\%). Although in all of the three risk stratification systems it was the highest risk group in which most TE events occured. (Landolfi: 44,9\%, IPSET: 39,0\%, R-IPSET: $46,0 \%)$. Also, in every system we found significant difference in the thrombosis-free survival between patients in the highest risk group and those in the prior risk groups (Landolfi: $p=0.032$, IPSET: p<0.001, R-IPSET: $\mathrm{p}<0.001$ ).

The most sensitive indicator of thrombosis after diagnosis is the high-risk group of the IPSET system (88.2\%). The negative predictive value is also the highest in the IPSET stratification $(88.2 \%)$. As for specificity and positive predictive value the R-IPSET system turned out to be the best. The Landolfi stratification is a good theoretical model, but in practice it seemed to be less usable than the other systems. It also has the disadvantage of using fewer objective parameters (such as smoking) that are based upon questioning of the patients. Taking everything into consideration the R-IPSET system seems more balanced than the others, with the sum of percentages being the highest. In our study it proved to be the strongest model (Table 4).

Table 4: Reliability of Landolfi-, IPSET and R-IPSET thrombosis risk models.

\begin{tabular}{|c|c|c|c|}
\hline & Landolfi (Very High) & IPSET (High) & R-IPSET (High) \\
\hline Sensitivity & $76,3 \%$ & $88,2 \%$ & $76,3 \%$ \\
\hline Specificity & $32,9 \%$ & $34,8 \%$ & $57,8 \%$ \\
\hline Positive predictive value & $34,9 \%$ & $39,0 \%$ & $46,0 \%$ \\
\hline Negative predictive value & $74,6 \%$ & $86,2 \%$ & $83,8 \%$ \\
\hline
\end{tabular}

\section{CALR positive cases}

Out of the 237 ET patients CALR mutation was detected in 45 cases. Male dominance and lower median age at diagnosis was observed among CALR patients, but these differences were not significant (Table 5). There was no difference between CALR positive and negative cases regarding the frequency of disease progression and the ratio of fatal outcomes, however, we did find significant difference between the two groups in terms of time to death/disease progression. Patients with CALR mutation had longer progression free- $(p=0,017)$ and overall survival $(p=0,021)$. CALR mutation negative patients suffered TE complications more often $(34,4 \%$ vs. $22,2 \%)$, although the difference was not significant $(\mathrm{p}=0,116)$.

\section{Discussion}

Thromboembolic complications are the major cause of morbidity and mortality of ET patients. Because of the favourable prognosis of the disease the most important aspect of the treatment is the prevention of TE events. Using thrombosis risk stratification systems, we can categorize patients into different risk groups and choose the most appropriate therapeutic option. We analysed the data of 237 patients from the "HUMYPRON” registry with an average follow-up period of 10 years. After diagnosis 76 patients (32.1\%) had TE event, while bleeding complication was observed in 8 cases (3.4\%). While before diagnosis the major arterial events were the most common TE complications, after diagnosis the minor arterial events occurred most often. Thus, applied therapy seems to be more protective against major arterial episodes.

When testing Landolfi-, IPSET- and R-IPSET risk stratifications on our sample collection, we found significant difference in the thrombosis-free survival of high risk and low risk patients in all three models (Landolfi: $p=0.032$, IPSET: $p<0.001$, R-IPSET: $\mathrm{p}<0.001$ ). On the other hand, the incidence of TE events was lower in the intermediate risk groups than in low-risk groups. The discrepancy between of our findings and literature data may be due to the differences in event definition, patient selection and applied therapy.

R-IPSET system proved to be the strongest model on our sample set. Landolfi risk stratification turned out to be the least applicable, which might be explained, along with the differences mentioned above, with the insufficiency of our data, since it strongly relies on questioning the patients.

It is known that patients with CALR mutation can expect better prognosis [18]. A study with a median follow-up of almost 13 years found that CALR positive cases are associated with younger age, male dominance and less TE complications [19]. Our results are similar, though the differences were not significant. However, we found significant difference in progression free- $(p=0,017)$ and overall survival $(p=0,021)$ between CALR positive and negative cases (Table 5). 
Table 5: Evaluating the presence of CALR mutation.

\begin{tabular}{|c|c|c|c|}
\hline \multirow{2}{*}{ Event } & \multicolumn{2}{|c|}{ CALR Mutation } & \multirow{2}{*}{$\mathbf{p}$} \\
\hline & yes $(\mathrm{N}=45)$ & no $(\mathrm{N}=192)$ & \\
\hline \multicolumn{3}{|l|}{ Gender, n (\%) } & \multirow{3}{*}{$\mathrm{p}=0,232$} \\
\hline male & $18(40,0)$ & $59(30,7)$ & \\
\hline female & $27(60,0)$ & $133(69,3)$ & \\
\hline \multicolumn{3}{|l|}{ Age at the diagnosis, years } & \multirow{4}{*}{$\mathrm{p}=0,651$} \\
\hline median & 61 & 62 & \\
\hline minimum-maximum & $27-81$ & $25-92$ & \\
\hline average $+/-$ SD & $60,1+/-13,5$ & $61,1+/-13,5$ & \\
\hline \multicolumn{3}{|l|}{ Death, n (\%) } & \multirow{3}{*}{$\mathrm{p}=0,549$} \\
\hline yes & $10(22,2)$ & $51(26,6)$ & \\
\hline no & $35(77,8)$ & $141(73,4)$ & \\
\hline \multicolumn{3}{|c|}{ Time to death/length of follow up, years } & \multirow{4}{*}{$\mathrm{p}=0,021$} \\
\hline median & 11 & 8 & \\
\hline minimum-maximum & $1-27$ & $1-27$ & \\
\hline average +/- SD & $11+/-6,8$ & $8,5+/-5,5$ & \\
\hline \multicolumn{3}{|l|}{ Progression, n (\%) } & \multirow{3}{*}{$\mathrm{p}=0,899$} \\
\hline yes & $6(13,3)$ & $27(14,1)$ & \\
\hline no & $39(86,7)$ & $165(85,9)$ & \\
\hline \multicolumn{3}{|c|}{ Time to progression/length of follow up, years } & \multirow{4}{*}{$\mathrm{p}=0,017$} \\
\hline median & 13 & 7 & \\
\hline minimum-maximum & $6-14$ & $2-14$ & \\
\hline average +/- SD & $11,5+/-3,3$ & $7,4+/-3,5$ & \\
\hline \multicolumn{3}{|l|}{ TE event, n (\%) } & \multirow{3}{*}{$\mathrm{p}=0,116$} \\
\hline yes & $10(22,2)$ & $66(34,4)$ & \\
\hline no & $35(77,8)$ & $126(65,6)$ & \\
\hline
\end{tabular}

Examining potential thrombotic risk factors, we found that only prior thrombotic event shows significant correlation $(p<0.001)$ with TE event after diagnosis. The independent and strongly significant effect of earlier thrombosis on post-diagnostic TE complications anticipates the presence of a prothrombotic phenomenon. Investigations of the last few years revealed that CHIP (clonal haematopoiesis of indeterminate potential) is an independent risk factor of the vascular events. [20,21]. According to literature data the susceptibility of CHIP patients to vascular complications is due to non-driver mutations (ASXL1, TET2, DNMT3A, JAK2) observed in clonal haematopoesis [22-24]. As many of the patients had suffered thrombotic episodes years before the diagnosis it can be presumed that some of the pre-diagnostic TE events may have manifested in the CHIP fase (Figure 1). According to our data we suggest that the TE events observed in ET patients are actually attributes of CHIP and not ET itself. The LCN2 (lypocalin-2) and MMP9 (matrix metalloproteinase-9) produced by neutrophil granulocytes/ monocytes arising from clonal haematopoesis damage the endothel, leading to increased thrombotic risk. [25].

\section{Conclusion}

Analyzing data of 237 ET patients of the „HUMYPRON” registry we showed that R-IPSET thrombosis risk system was the most applicable to our sample set. Univariate analysis revealed that thrombotic events before diagnosis have an independent, strongly significant effect on post-diagnostic TE episodes $(\mathrm{p}<0.001)$. We suppose that this may be the result of CHIP, and so, patients acquire predisposition to thrombotic complications due to clonal haematopoesis before the actual evolution of ET.

\section{References}

1. Ma X, Vanasse G, Cartmel B, Wang Y, Selinger HA (2008) Prevalence of polycythemia vera and essential thrombocythemia. American Journal of Hematology 83(5): 359-362.

2. Johansson P (2006) Epidemiology of the myeloproliferative disorder's polycythemia vera and essential thrombocythemia. Seminars in Thrombosis and Hemostasis 32(3): 171-173.

3. Cortelazzo S, Viero P, Finazzi GA, D’Emilio A, Rodeghiero F, et al. (1990) Incidence and risk factors for thrombotic complications in a historical 
cohort of 100 patients with essential thrombocythemia. Journal of Clinical Oncology 8(3): 556-562.

4. Arber DA, Orazi A, Hasserjian R, Thiele J, Borowitz MJ, et al. (2016) The 2016 revision to the World Health Organization classification of myeloid neoplasms and acute leukemia. Blood 127(20): 2391-2405.

5. Barbui T, Thiele J, Gisslinger H, Finazzi G, Vannucchi AM, et al. (2016) The 2016 revision of WHO classification of myeloproliferative neoplasms: Clinical and molecular advances. Blood Reviews 30(6): 453-459.

6. Tefferi A (2016) Myeloproliferative neoplasms: A decade of discoveries and treatment advances. American Journal of Hematology 91(1): 50-58.

7. Tefferi A, Barbui $T$ (2017) Polycythemia vera and essential thrombocythemia: 2017 update on diagnosis, risk-stratification, and management. American Journal of Hematology 92(1): 94-108.

8. Cerquozzi S, Tefferi A (2015) Blast transformation and fibrotic progression in polycythemia vera and essential thrombocythemia: A literature review of incidence and risk factors. Blood Cancer Journal 5(11): e366.

9. Cortelazzo S, Viero P, Finazzi GA, D’Emilio A, Rodeghiero F, et al. (1990) Incidence and risk factors for thrombotic complications in a historical cohort of 100 patients with essential thrombocythemia. Journal of Clinical Oncology 8(3): 556-562.

10. Jensen MK, de Nully Brown P, Nielsen OJ, Hasselbalch HC (2000) Incidence, clinical features and outcome of essential thrombocythaemia in a well-defined geographical area. European Journal of Haematology 65(2): 132-139.

11. Barbui T, Vannucchi AM, Buxhofer-Ausch V, De Stefano V, Betti S, et al. (2015) Practice-relevant revision of IPSET-thrombosis based on 1019 patients with WHO-defined essential thrombocythemia. Blood Cancer J 5(11): e369.

12. Landolfi R, Gennaro L (2008) Prevention of thrombosis in polycythemia vera and essential thrombocythemia. Haematologica 93(3): 331-335.

13. Tefferi A, Vannucchi AM, Barbui T (2018) Essential thrombocythemia treatment algorithm 2018. Blood Cancer Journal 8(1): 1-6.

14. Dombi P, Illés Á, Demeter J, Homor L, Simon Z, et al. (2017) Anagrelide reduces thrombotic risk in essential thrombocythaemia $v s$. hydroxyurea plus aspirin. European Journal of Haematology 98(2): 106-111.
15. Dombi P, Illés Á, Demeter J, Homor L, Simon Z, et al. (2016) Development of the registry for Philadelphia-negative chronic myeloproliferative neoplasia in Hungary. Orvosi Hetilap 157(3): 98-103.

16. Gisslinger H, Gotic M, Holowiecki J, Penka M, Thiele J, et al. (2013) Anagrelide compared with hydroxyurea in WHO-classified essential thrombocythemia: The Anahydret Study, a randomized controlled trial. Blood 121(10): 1720-1728.

17. Kellner A, Dombi P, Illes A, Demeter J, Homor L, et al. (2020) Anagrelide influences thrombotic risk, and prolongs progression-free and overall survival in essential thrombocythaemia vs. hydroxyurea plus aspirin. European Journal of Haematology 105(4): 408-418.

18. Carla AS, Van Obbergh, Billiet J, Lierman E, Timothy D, et al. (2015) Analysis of phenotype and outcome in essential thrombocythemia with CALR or JAK2 mutations. Haematologica 100(7): 893-897.

19. Tefferi, Ayalew EA, Lasho TL, Belachew AA, Ketterling RP, et al. (2014) Calreticulin mutations and long-term survival in essential thrombocythemia. Leukemia 28(12): 2300-2303.

20. Jaiswal S, Fontanillas P, Flannick J, Manning A, Grauman PV, et al. (2014) Age-related clonal hematopoiesis associated with adverse outcomes. New England Journal of Medicine 371(26): 2488-2498.

21. Libby P, Ebert BL (2018) CHIP (clonal hematopoiesis of indeterminate potential) potent and newly recognized contributor to cardiovascular risk. Circulation 138(7): 666-668.

22. Jaiswal S, Natarajan P, Silver AJ, Gibson CJ, Bick AG, et al. (2017) Clonal hematopoiesis and risk of atherosclerotic cardiovascular disease. New England Journal of Medicine 377(2): 111-121.

23. Steensma DP (2018) Clinical consequences of clonal hematopoiesis of indeterminate potential. Hematology 2(22): 3404-3410.

24. Buscarlet M, Provost S, Zada YF, Bourgoin V, Mollica L, et al. (2018) Lineage restriction analyses in CHIP indicate myeloid bias for $\mathrm{TET}_{2}$ and multipotent stem cell origin for $\mathrm{DNMT}_{3} \mathrm{~A}$. Blood 132(3): 277-280.

25. Rajnics P, Kellner Á, Karádi É, Moizs M, Bödör C, et al. (2016) Increased Lipocalin 2 level may have important role in thrombotic events in patients with polycythemia vera and essential thrombocythemia. Leukemia Research 48: 101-106. 